\title{
Simple Expressions for the Correlation between Fading Channel Error Rates
}

\author{
Alan Clark, Desmond Taylor \& Peter Smith \\ Dept. Electrical and Computer Engineering \\ University of Canterbury \\ Private Bag 4800 \\ Christchurch, New Zealand \\ Email: \{a.clark,d.taylor,p.smith\}@elec.canterbury.ac.nz
}

\begin{abstract}
Given digital transmission over two correlated Rician fading channels, we present a readily calculable expression for a tight approximation to the correlation between the probabilities of symbol error on each channel. For the Rayleigh fading channel the expression is exact. This correlation expression has applications in error performance analysis of fading systems, particularly multichannel systems and Markov modelling of error events. As an example we use these expressions to bound the block error rate of an OFDM system.
\end{abstract}

\section{INTRODUCTION AND PRELIMINARIES}

Error performance analysis of systems employing space, time or frequency diversity often requires calculation of the correlation between symbol error rates for transmission over correlated fading channels, as discussed in [1]. Systems where transmission is affected by correlated channel gains include modulation over a fading channel, OFDM and MIMO. Knowledge of the symbol error rate correlation is essential for Markov modelling of the error process [2], as in the analysis of [3], as well as in the analysis of error correcting codes for fading channels. In this paper we present closed form representations for a tight upper bound on the correlation between the symbol error probabilities of BPSK transmission over correlated Rician fading channels. In addition, for Rayleigh fading channels we obtain an exact series representation of the correlation. We then demonstrate a simple application of these expressions by calculating a lower bound on the block error rate of an OFDM system.

We assume transmission of BPSK symbols with energy $E_{0}$, bandlimited noise of power spectral density $N_{0}$, and a slow fading channel response magnitude $|H|$ that remains constant over each symbol duration. Given a maximum likelihood receiver with perfect channel state information, the probability of symbol error is well known to be

$$
P=\frac{1}{2} \operatorname{erfc}\left(\sqrt{|H|^{2} \frac{E_{0}}{N_{0}}}\right) .
$$

For a Rician or Rayleigh fading channel $|H|^{2}$ follows a non-central chi-squared [4] or an exponential [5] distribution respectively. Note that for the Rician channel we may use the $\operatorname{erfc}(\cdot)$ form of [6] to write the mean error rate $\mathbf{E}[P]$ as [7]

$$
\frac{1}{\pi} \int_{0}^{\frac{\pi}{2}} \frac{(K+1) \sin ^{2} \theta}{\gamma_{0}+(K+1) \sin ^{2} \theta} \exp \left(\frac{-K \gamma_{0}}{\gamma_{0}+(K+1) \sin ^{2} \theta}\right) d \theta
$$

where $K$ is the Rice factor and $\gamma_{0}=\frac{E_{0}}{N_{0}}$. For the Rayleigh fading case $(K=0)$ this simplifies [7] to

$$
\mathbf{E}[P]=\frac{1}{2}\left(1-\sqrt{\frac{\gamma_{0}}{1+\gamma_{0}}}\right) .
$$

In this paper we find simple closed form approximations and series representations for $\mathbf{E}\left[P_{1} P_{2}\right]$, where $P_{1}$ and $P_{2}$ are the error probabilities for two correlated Rician or Rayleigh fading channels with gains denoted $\left|H_{1}\right|^{2}$ and $\left|H_{2}\right|^{2}$. We impose no restriction on the coefficient of correlation $\rho$ between the channel gains. This allows us to compute the correlation between error rates at different frequencies, locations or times. In the next section we outline the calculation of closed form expressions for $\mathbf{E}\left[P_{1} P_{2}\right]$. In Section III we illustrate their use in bounding the block error rate of an OFDM system, and compare this to simulations. Section IV concludes this paper.

\section{CORRELATION ExPRESSIONS}

\section{A. Rician Channels}

When both channels experience Rician fading, the joint pdf of $\left|H_{1}\right|^{2}$ and $\left|H_{2}\right|^{2}$ is [4]

$$
\begin{aligned}
& f_{\left|H_{1}\right|^{2},\left|H_{1}\right|^{2}}(x, y) \\
= & \frac{1}{1-\rho^{2}} \exp \left(-\frac{x+y-2 a^{2}(1-\rho)}{1-\rho^{2}}\right) \sum_{k=0}^{\infty}(-1)^{k} k(k-1) \\
& \times \mathrm{I}_{\mathrm{k}}(-2 \rho \sqrt{x y}) \mathrm{I}_{\mathrm{k}}\left(\frac{2 a(1-\rho) \sqrt{x}}{1-\rho^{2}}\right) \mathrm{I}_{\mathrm{k}}\left(\frac{2 a(1-\rho) \sqrt{y}}{1-\rho^{2}}\right)
\end{aligned}
$$

where we have assumed $\mathbf{E}\left[\left|H_{1}\right|^{2}\right]=\mathbf{E}\left[\left|H_{2}\right|^{2}\right]=a+1$ and the Rice factor is $K=a^{2}$. We may then write the correlation $\mathbf{E}\left[P_{1} P_{2}\right]$ as

$\frac{1}{4} \int_{0}^{\infty} \int_{0}^{\infty} \operatorname{erfc}\left(\sqrt{\gamma_{0} x}\right) \operatorname{erfc}\left(\sqrt{\gamma_{0} y}\right) f_{\left|H_{1}\right|^{2},\left|H_{1}\right|^{2}}(x, y) d x d y$

Exact calculation of this expression appears intractable. 
We decompose the channel gains $H_{1}$ and $H_{2}$ into the underlying Gaussian random variables. That is, $H_{1}=a+$ $X_{1}+j Y_{1}$ and $H_{2}=a+X_{2}+j Y_{2}$, where we assume that both channels have the same line of sight component $a$, and $X_{1}$, $Y_{1}, X_{2}$ and $Y_{2}$ are identically distributed zero mean Gaussian random variables. Without loss of generality we may assume $\mathbf{E}\left[X_{1}^{2}\right]=\frac{1}{2}$, so that $\mathbf{E}\left[\left|H_{1}\right|^{2}\right]=\mathbf{E}\left[\left|H_{2}\right|^{2}\right]=a+1$. We may then write

$$
\begin{aligned}
H_{2} & =a+\tilde{X}+j \tilde{Y}, \text { where } \\
\tilde{X} & =\rho U_{1}+\sqrt{1-\rho^{2}} U_{2}, \quad \tilde{Y}=\rho V_{1}+\sqrt{1-\rho^{2}} V_{2}
\end{aligned}
$$

so that $U_{1}, U_{2}, V_{1}$ and $V_{2}$ are zero mean independent and identically distributed Gaussian random variables with variance $\frac{1}{2}$.

Next, recognise [8] that $P \leq \frac{1}{2} \exp \left(-\gamma_{0}|H|^{2}\right)$, which is a tight upper bound at high SNR. We may then bound (5) as

$$
\begin{aligned}
\mathbf{E}\left[P_{1} P_{2}\right] & \leq \frac{1}{4} \mathbf{E}\left[\exp \left(-\gamma_{0}\left|H_{1}\right|^{2}\right) \exp \left(-\gamma_{0}\left|H_{2}\right|^{2}\right)\right] \\
& =\frac{1}{4} \mathrm{~g}_{1}(a) \mathrm{g}_{1}(0) .
\end{aligned}
$$

We have thus implicitly defined $g_{1}(z)$ to be

$$
\begin{aligned}
& \int_{-\infty}^{\infty} \int_{-\infty}^{\infty} \exp \left(-\gamma_{0}\left[(x+z)^{2}+\left(\rho x+\sqrt{1-\rho^{2}} y+z\right)^{2}\right]\right) \\
& \quad \times f_{U_{1}}(x) f_{U_{2}}(y) d x d y \\
& =\frac{1}{\pi} \int_{-\infty}^{\infty} \exp \left(-\gamma_{0}\left[\left(1-\rho^{2}\right) y^{2}+2 \sqrt{1+\rho^{2}} z y+2 z^{2}\right]\right) \\
& \quad \times \exp \left(-y^{2}\right) \mathrm{g}_{2}(y, z) f_{U_{1}}(x) f_{U_{2}}(y) d x d y
\end{aligned}
$$

Furthermore,

$$
\begin{aligned}
\mathrm{g}_{2}(y, z) \triangleq \int_{-\infty}^{\infty} & \exp \left(-\gamma_{0}\left[\left(1+\rho^{2}\right) x^{2}\right.\right. \\
& \left.\left.+2(1+\rho) z x+2 \rho \sqrt{1-\rho^{2}} y x\right]-x^{2}\right) d x .
\end{aligned}
$$

We define the constants $C_{1}=1+\gamma_{0}\left(1+\rho^{2}\right), C_{2}=$ $\frac{1}{\sqrt{C_{1}}} \gamma_{0} \rho \sqrt{1-\rho^{2}}$, and let $\mathrm{g}_{3}(z)=\frac{1}{\sqrt{C_{1}}} \gamma_{0}(1+\rho) z$. After completing the square and rearranging the integrand in (9) to the form of a Gaussian pdf, we may write

$$
\mathrm{g}_{2}(y, z)=\sqrt{\frac{\pi}{C_{1}}} \exp \left(\left[\mathrm{g}_{3}(z)+C_{2} y\right]^{2}\right) .
$$

We substitute (10) into (8) to obtain

$$
\begin{aligned}
\mathrm{g}_{1}(z)= & \sqrt{\frac{1}{\pi C_{1}}} \int_{-\infty}^{\infty} \exp \left(-\gamma_{0}\left[\left(1-\rho^{2}\right) y^{2}+2 \sqrt{1-\rho^{2}} z y\right.\right. \\
& \left.\left.+2 z^{2}\right]-y^{2}\right) \exp \left(\left[\mathrm{g}_{3}(z)+C_{2} y\right]^{2}\right) d y \\
= & \frac{1}{\sqrt{C_{1} C_{3}}} \exp \left(\frac{\mathrm{g}_{4}^{2}(z)}{4 C_{3}}-\mathrm{g}_{5}(z)\right)
\end{aligned}
$$

where we have again completed the square, arranged the integrand into the form of a Gaussian pdf and set

$$
\begin{aligned}
C_{3} & =\gamma_{0}\left(1-\rho^{2}\right)+C_{2}^{2} \\
\mathrm{~g}_{4}(z) & =2 \gamma_{0} \sqrt{1-\rho^{2}} z+2 C_{2} \mathrm{~g}_{3}(z) \\
\mathrm{g}_{5}(z) & =2 \gamma_{0} z+\mathrm{g}_{3}^{2}(z) .
\end{aligned}
$$

Finally, substituting (11) into (7) we obtain

$$
\begin{aligned}
\mathbf{E}\left[P_{1} P_{2}\right] \leq & \frac{1}{4} \frac{1}{C_{1} C_{3}} \exp \left(\frac{\mathrm{g}_{4}^{2}(a)}{4 C_{3}}-\mathrm{g}_{5}^{2}(a)\right) \\
& \times \exp \left(\frac{\mathrm{g}_{4}^{2}(0)}{4 C_{3}}-\mathrm{g}_{5}^{2}(0)\right) \\
= & \frac{1}{4} \frac{1}{C_{1} C_{3}} \exp \left(\frac{\mathrm{g}_{4}^{2}(a)}{4 C_{3}}-\mathrm{g}_{5}^{2}(a)\right)
\end{aligned}
$$

since $\mathrm{g}_{4}(0)=\mathrm{g}_{5}(0)=0$. We therefore have a readily calculable upper bound on the correlation between the probabilities of BPSK symbol error on two Rician fading channels.

\section{B. Rayleigh Channels}

For two Rayleigh fading channels $f_{\left|H_{1}\right|^{2},\left|H_{2}\right|^{2}}(x, y)$ is a bivariate correlated exponential density function [5]. Substituting this into (5) we obtain

$$
\begin{gathered}
\quad \mathbf{E}\left[P_{1} P_{2}\right] \\
=\frac{1}{4} \int_{0}^{\infty} \int_{0}^{\infty} \operatorname{erfc}\left(\sqrt{\gamma_{0} x}\right) \operatorname{erfc}\left(\sqrt{\gamma_{0} y}\right) f_{\left|H_{1}\right|^{2},\left|H_{2}\right|^{2}}(x, y) d x d y \\
=\frac{1}{4} \int_{0}^{\infty} \int_{0}^{\infty} \frac{1}{1-\rho^{2}} \operatorname{erfc}\left(\sqrt{\gamma_{0} x}\right) \operatorname{erfc}\left(\sqrt{\gamma_{0} y}\right) \exp \left(-\frac{x+y}{1-\rho^{2}}\right) \\
\quad \times \mathrm{I}_{0}\left(\frac{2 \rho \sqrt{x y}}{1-\rho^{2}}\right) d x d y, \text { for } 0 \leq \rho<1 .
\end{gathered}
$$

This expression contains the integral

$$
\begin{aligned}
& \mathrm{h}_{1}(y)=\int_{0}^{\infty} \operatorname{erfc}\left(\sqrt{\gamma_{0} x}\right) \exp \left(\frac{-x}{1-\rho^{2}}\right) \mathrm{I}_{0}\left(\frac{2 \rho \sqrt{x y}}{1-\rho^{2}}\right) d x \\
& =\sum_{i=0}^{\infty} \frac{1}{(i !)^{2}} \frac{\rho^{2 i} y^{i}}{\left(1-\rho^{2}\right)^{2 i}} \int_{0}^{\infty} \operatorname{erfc}\left(\sqrt{\gamma_{0} x}\right) \exp \left(\frac{-x}{1-\rho^{2}}\right) x^{i} d x
\end{aligned}
$$

using a Bessel function series expansion [9]. A general term of this summation contains the integral

$$
\begin{aligned}
\mathrm{h}_{2}(i)= & \int_{0}^{\infty} \operatorname{erfc}\left(\sqrt{\gamma_{0} x}\right) \exp \left(-\frac{x}{1-\rho^{2}}\right) x^{i} d x \\
= & 2 i !\left(1-\rho^{2}\right)^{i+1}\left[\frac{1}{2}\left(1-\sqrt{\frac{\gamma_{0}\left(1-\rho^{2}\right)}{2+\gamma_{0}\left(1-\rho^{2}\right)}}\right)\right]^{i+1} \\
& \times \sum_{n=0}^{i}\left(\begin{array}{c}
i+n \\
n
\end{array}\right)\left[\frac{1}{2}\left(1+\sqrt{\frac{\gamma_{0}\left(1-\rho^{2}\right)}{2+\gamma_{0}\left(1-\rho^{2}\right)}}\right)\right]^{n}
\end{aligned}
$$

after some manipulation and using a result from [8, sec. 14]. We then substitute $h_{2}(i)$ into $h_{1}(y)$ and this result into (14) 
to write

$$
\begin{aligned}
\mathbf{E}\left[P_{1} P_{2}\right]= & \frac{1}{4} \frac{1}{1-\rho^{2}} \sum_{i=0}^{\infty} \frac{1}{(i !)^{2}} \mathrm{~h}_{2}(i)\left[\frac{\rho^{2}}{\left(1-\rho^{2}\right)^{2}}\right]^{i} \\
& \times \int_{0}^{\infty} \operatorname{erfc}\left(\sqrt{\gamma_{0} y}\right) \exp \left(-\frac{y}{1-\rho^{2}}\right) y^{i} d y
\end{aligned}
$$

Finally, recognising that the remaining integral in (17) is equal to $\mathrm{h}_{2}(i)$, we may write

$$
\mathbf{E}\left[P_{1} P_{2}\right]=\frac{1}{4\left(1-\rho^{2}\right)} \sum_{i=0}^{\infty} \frac{1}{(i !)^{2}}\left[\frac{\rho^{2}}{\left(1-\rho^{2}\right)^{2}}\right]^{i}\left[\mathrm{~h}_{2}(i)\right]^{2}
$$

This expression allows simple numerical calculation of $\mathbf{E}\left[P_{1} P_{2}\right]$, since it may be shown to be rapidly convergent for $0 \leq \rho<1$.

Equations (18) and (13) are the key results of this paper, giving simple series representations for the correlation between symbol error probabilities for the Rayleigh fading channel and an upper bound on the correlation between symbol error probabilities for the Rician fading channel, respectively. These expressions may be used in the analysis of multichannel systems. In the following section we give a brief example of such an application.

\section{EXAMPLE: OFDM BLOCK ERROR RATE}

As an example we now apply the correlations of (18) and (13) to calculate a lower bound on the block error rate of an $N$ subcarrier OFDM system transmitting over Rician and Rayleigh fading channels. During the $n^{\text {th }}$ time interval BPSK subcarrier symbols $\mathbf{S}_{n}=\left\{S_{n, 1}, S_{n, 2}, \ldots, S_{n, N}\right\}$ are transmitted, with $S_{n, k} \in\left\{-\sqrt{E_{0}},+\sqrt{E_{0}}\right\}$.

We assume ideal channel state information, time synchronisation such that intersymbol interference is negligible and frequency synchronisation such that subcarrier orthogonality is preserved. Furthermore, we assume perturbation by additive white Gaussian noise with power spectral density $N_{0}$. At the receiver we obtain a noise corrupted version of the transmitted symbol $\mathbf{R}_{n}=\left\{R_{n, 1}, R_{n, 2}, \ldots, R_{n, N}\right\}$, where $R_{n, k}=H_{n, k} S_{n, k}+N_{n, k}$, and $N_{n, k}$ is a zero mean complex Gaussian random variable with variance $\frac{N_{0}}{2}$ per dimension. We then acquire an estimate $\hat{\mathbf{S}}_{n}=\left\{\hat{S}_{n, 1}, \hat{S}_{n, 2}, \ldots, \hat{S}_{n, N}\right\}$ of the transmitted vector. The probability $P_{n, k}$ that $\hat{S}_{n, k}$ is incorrectly estimated is

$$
P_{n, k} \triangleq \operatorname{Pr}\left(\hat{S}_{n, k} \neq S_{n, k}\right)=\frac{1}{2} \operatorname{erfc}\left(\sqrt{\gamma_{0}\left|H_{n, k}\right|^{2}}\right) .
$$

An OFDM block error occurs if one or more of the transmitted symbols $S_{n, k}, k=1, \ldots, N$, is incorrectly estimated, so that $\hat{\mathbf{S}}_{n} \neq \mathbf{S}_{n}$, and we denote this event $B_{n}$. Averaging in time over each subchannel gain and using the principle of inclusion and exclusion [10] we may write the mean block error rate as

$$
\begin{aligned}
\mathbf{E}\left[\operatorname{Pr}\left(B_{n}\right)\right]= & \sum_{k=1}^{N} \mathbf{E}\left[P_{n, k}\right]-\sum_{\substack{k_{2}>k_{1} \\
k_{1}, k_{2}=1}}^{N} \mathbf{E}\left[P_{n, k_{1}} P_{n, k_{2}}\right] \\
& +\sum_{\substack{k_{3}>k_{2}>k_{1} \\
k_{1}, k_{2}, k_{3}=1}}^{N} \mathbf{E}\left[P_{n, k_{1}} P_{n, k_{2}} P_{n, k_{3}}\right]-\ldots+\ldots
\end{aligned}
$$

We may then write bounds on the mean block error rate as

$$
\begin{aligned}
& \mathbf{E}\left[\operatorname{Pr}\left(B_{n}\right)\right] \leq \min \left\{\sum_{k=1}^{N} \mathbf{E}\left[P_{n, k}\right], 1\right\} \\
& \mathbf{E}\left[\operatorname{Pr}\left(B_{n}\right)\right] \geq \max \left\{\sum_{k=1}^{N} \mathbf{E}\left[P_{n, k}\right]-\sum_{\substack{k_{2}>k_{1} \\
k_{1}, k_{2}=1}}^{N} \mathbf{E}\left[P_{n, k_{1}} P_{n, k_{2}}\right], 0\right\} .
\end{aligned}
$$

The upper bound is the well known union bound and is readily calculable using expressions (2) and (3) for the Rician and Rayleigh fading channels respectively. The lower bound may be calculated for the Rician channel by using the bound of (13), and for the Rayleigh channel with the series representation of (18). Note that tighter upper and lower bounds may be obtained by incorporating higher order terms of (20). However, closed form expressions for the integrals required to calculate these terms appear intractable.

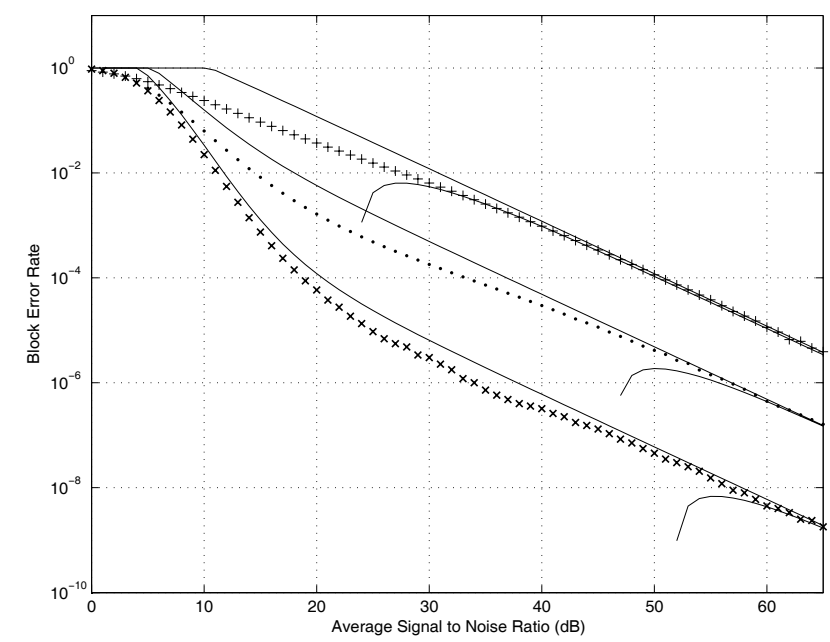

Fig. 1. Analytical Bounds (solid lines) and Simulated $(+, \cdot, \times)$ OFDM Block Error Rate for a Rayleigh fading channel $(+)$, Rician channel with $K=5$ $(\cdot)$ and $K=10(\times)$.

We calculate upper and lower bounds on the block error rate for a 48 subcarrier OFDM system transmitting over a Rician fading channel for Rice factors $K=5$ and $K=10$, and for the Rayleigh fading channel. We presume the channel specular component is described by a Jakes' model [11] and an exponential power delay profile with rms excess delay $\tau_{\text {rms }}=$ $15 \mathrm{~ns}$. The subcarrier frequency separation is $\Delta f=1.7 \mathrm{MHz}$, 
and the correlation coefficient between the $k_{1}^{\text {th }}$ and $k_{2}^{\text {th }}$ channel gains, $\left|H_{n, k_{1}}\right|^{2}$ and $\left|H_{n, k_{2}}\right|^{2}$, is [11]

$$
\rho=\left[1+\left(2 \pi \tau_{\mathrm{rms}} \Delta f\left|k_{2}-k_{1}\right|\right)^{2}\right]^{-1} .
$$

We simulate this system, presuming a receiver velocity of $5 \mathrm{~m} / \mathrm{s}$, and plot the simulated block error rate with the analytical block error rate bounds in Figure 1. At low SNR the lower bound is 0 , since the correlation sum in (21) is larger than the sum of means. Observe that the calculated bounds are tight at moderate and high SNR.

\section{Discussion}

We have derived a simple, easily evaluated series representation for the correlation between the probability of BPSK symbol error for two correlated Rayleigh fading channels. For Rician channels we have developed a simple, closed form upper bound on this correlation. We may apply a similar approach to find an upper bound on this correlation when QAM symbols are transmitted.

The correlations are useful for error performance analysis of systems on correlated fading channels, such as OFDM systems, MIMO systems or non-ideally interleaved fading systems. Furthermore, Markov modelling of error events requires the correlation between the probabilities of symbol errors. We have illustrated an application with an example: the calculation of a lower bound on the OFDM block error rate.

\section{ACKNOWLEDGMENT}

This work was supported in part by the Foundation for Research Science and Technology, New Zealand.

\section{REFERENCES}

[1] M. Zorzi and R. R. Rao, "Perspectives on the impact of error statistics on protocols for wireless networks," IEEE Personal Communications, vol. 6, no. 5, pp. 32-40, 1999.

[2] L. Kanal and A. Sastry, "Models for channels with memory and their applications to control," Proc. IEEE, vol. 66, pp. 724-744, July 1978.

[3] J. R. Yee and E. J. Weldon, "Evaluation of the performance of errorcorrecting codes on a Gilbert channel," IEEE Trans. Commun., vol. 43, no. 8, pp. 2316-2323, Aug. 1995.

[4] K. Miller, R. Bernstein, and L.E.Blumenson, "Generalized Rayleigh processes," Quarterly of Applied Mathematics, vol. 16, no. 2, pp. 137$145,1958$.

[5] R. K. Mallik, "On multivariate Rayleigh and exponential distributions," IEEE Trans. Inform. Theory, vol. 49, no. 6, pp. 1499-1515, June 2003.

[6] J. Craig, "A new, simple and exact result for calculating the probability of error for two-dimensional signal constellations," in Proc. IEEE MILCOM'91 Conf. Rec., Boston, 1991, pp. 25.5.1-25.5.5.

[7] M. K. Simon and M.-S. Alouini, Digital Communication over Fading Channels. New York, NY: John Wiley, 2000.

[8] J. G. Proakis, Digital Communications. New York, NY: McGraw-Hill, 2001.

[9] M. Abramowitz and I. A. Stegun, Eds., Handbook of Mathematical Functions. New York, NY: Dover, 1965.

[10] S. Ross, A First Course in Probability. New York, NY: MacMillan College, 1994.

[11] W. C. Jakes, Microwave Mobile Communications. New York, NY: IEEE Press, 1994. 